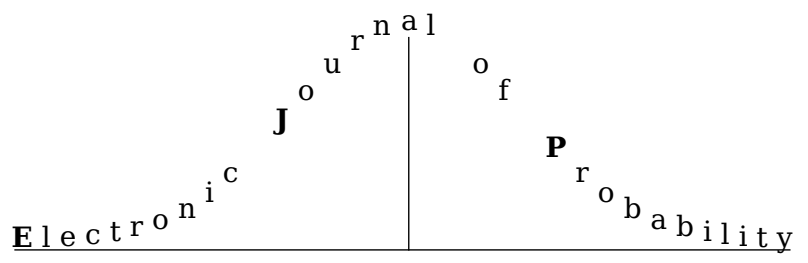

Electron. J. Probab. 25 (2020), article no. 97, 1-17.

ISSN: 1083-6489 https://doi.org/10.1214/20-EJP501

\title{
Radial processes for sub-Riemannian Brownian motions and applications*
}

\author{
Fabrice Baudoin ${ }^{\dagger} \quad$ Erlend Grong $^{\ddagger} \quad$ Kazumasa Kuwada ${ }^{\S}$ \\ Robert Neel ${ }^{\pi} \quad$ Anton Thalmaier"l
}

\begin{abstract}
We study the radial part of sub-Riemannian Brownian motion in the context of totally geodesic foliations. Itô's formula is proved for the radial processes associated to Riemannian distances approximating the Riemannian one. We deduce very general stochastic completeness criteria for the sub-Riemannian Brownian motion. In the context of Sasakian foliations and H-type groups, one can push the analysis further, and taking advantage of the recently proved sub-Laplacian comparison theorems one can compare the radial processes for the sub-Riemannian distance to one-dimensional model diffusions. As a geometric application, we prove Cheng's type estimates for the Dirichlet eigenvalues of the sub-Riemannian metric balls, a result which seems to be new even in the Heisenberg group.
\end{abstract}

Keywords: sub-Riemannian Brownian motion; radial process; stochastic completeness; subLaplacian comparison theorem; Sasakian manifold; H-type group; Riemannian foliation.

MSC2020 subject classifications: $53 \mathrm{C} 17 ; 35 \mathrm{H} 20$; $58 \mathrm{~J} 65$.

Submitted to EJP on February 7, 2020, final version accepted on July 26, 2020.

Supersedes arXiv: 2002.02556.

\footnotetext{
*The first author has been supported in part by NSF Grants DMS-1660031, DMS DMS-1901315 and the Simons Foundation; the second author by project 249980/F20 of the Norwegian Research Council; the third author by JSPS Grant-in-Aid for Young Scientist (KAKENHI) 26707004; the fourth author by a grant from the Simons Foundation (\#524713, RN), and the fifth author by FNR Luxembourg: OPEN project GEOMREV O14/7628746.

Kazumasa Kuwada deceased on December 29, 2018.

${ }^{\dagger}$ Department of Mathematics, University of Connecticut, 341 Mansfield Road, Storrs, CT 06269-1009, USA.

E-mail: fabrice. baudoin@uconn.edu, https://math.uconn.edu/person/fabrice-baudoin

${ }^{\ddagger}$ University of Bergen, Department of Mathematics, P.O. Box 7803, 5020 Bergen, Norway.

E-mail: erlend.grong@uib.no, https://www.uib.no/en/persons/Erlend.Grong

$\S^{\S}$ Department of Mathematics, Graduate School of Science, Tohoku University, 980-8578, Sendai, Japan.

"Department of Mathematics, Lehigh University, Bethlehem, PA 18015-3174, USA.

E-mail: robert. neel@lehigh. edu, https://www.lehigh.edu/ rwn209

"Department of Mathematics, University of Luxembourg, L-4364 Esch-sur-Alzette, Luxembourg.

E-mail: anton.thalmaier@uni.lu, http://math.uni.lu/thalmaier
} 


\section{Introduction}

In the context of Riemannian manifolds the study of the radial part of Brownian motion yields new proofs and sheds new light on several well-known theorems of Riemannian geometry; see for instance the paper [20] and the book [19] for an overview. Our goal in the present paper is to extend those techniques to the context of sub-Riemannian manifolds. In the last few years, the study of Brownian motion on sub-Riemannian manifolds has attracted a lot of interest, see [6], [7], [14] and [26], and several applications to the study of heat semigroup gradient bounds and functional inequalities on pathspaces have been obtained. Despite those numerous works the probabilistic study of the radial part of the sub-Riemannian Brownian motion is not yet developed. Taking advantage of the sub-Laplacian comparison theorems recently proved in [9], it is now possible to pursue such a study.

In this paper we focus on two classes of sub-Riemannian manifolds. The first one is the class of sub-Riemannian manifolds whose horizontal distribution is the horizontal distribution of some Riemannian foliation with totally geodesic leaves. The sub-Riemannian geometry of such structures is by now well understood, thanks to the works [8], [16] and [17]. A key insight is to approximate the sub-Riemannian distance $d_{0}$ by a family of Riemannian distances $d_{\varepsilon}, \varepsilon>0$ which converges to $d_{0}$ as $\varepsilon \rightarrow 0$. The sub-Laplacian comparison theorems associated to $d_{\varepsilon}$ obtained in that context are very general but with the drawback that there is no limit when $\varepsilon \rightarrow 0$, meaning that we can not deduce a result for the sub-Laplacian of the sub-Riemannian distance itself. One of the main results we obtain for the radial processes is Theorem 3.1, giving its Itô formula. Let $\left(\xi_{t}\right)_{t \geq 0}$ be the sub-Riemannian Brownian motion on a Riemannian manifold equipped with a totally geodesic foliation, and let $\Delta_{\mathcal{H}}$ be the sub-Laplacian (see the next section for the precise definitions). Denote by $r_{\varepsilon}$ the $d_{\varepsilon}$-distance from a fixed point $x_{0}$ and by $\zeta$ the lifetime of the process. Then

$$
r_{\varepsilon}\left(\xi_{t \wedge \zeta}\right)=r_{\varepsilon}\left(\xi_{0}\right)+\beta_{t \wedge \zeta}^{\varepsilon}+\int_{0}^{t \wedge \zeta} \Delta_{\mathcal{H}} r_{\varepsilon}\left(\xi_{s}\right) d s-l_{t \wedge \zeta}^{\varepsilon}
$$

where $l_{t}^{\varepsilon}$ is a non-decreasing continuous process which increases only when $\xi_{t}$ is in the $d_{\varepsilon}$ cut-locus of $x_{0}$ and where $\beta_{t}^{\varepsilon}$ is a martingale on $\mathbb{R}$, starting from 0 , with $d\left\langle\beta^{\varepsilon}\right\rangle \leq 2 d t$. This decomposition is the sub-Riemannian analogue of Kendall's well-known result [22]. However, note that $\beta_{t}^{\varepsilon}$ in this result is not a Brownian motion, unlike in the Riemannian case. Even if $\beta_{t}^{\varepsilon}$ is not a Brownian motion, we are still able in Section 4 to prove very general stochastic completeness criteria, see Theorem 4.3.

The second class of sub-Riemannian manifolds we will focus on is the class of Sasakian foliations. Sasakian foliations are a special class of totally geodesic foliations for which the leaves have dimension one. In that particular class of examples, it was proved in $[9,23]$ that it is possible to prove sharp sub-Laplacian comparison theorems for $\Delta_{\mathcal{H}} r_{\varepsilon}$ which actually have a limit when $\varepsilon \rightarrow 0$. As a consequence, we are able to study the radial process with respect to the sub-Riemannian distance itself. Let $n$ be the dimension of the horizontal distribution. Our main result is the comparison Theorem 5.2. It states that under natural curvature lower bounds (expressed in terms of constants $k_{1}$ and $k_{2}$ ), one has in a weak sense,

$$
r_{0}\left(\xi_{t}\right) \leq \tilde{\xi}_{t}
$$

where $\tilde{\xi}$ is a one-dimensional diffusion with generator

$$
L_{k_{1}, k_{2}}=\left(F_{\mathrm{Sas}}\left(r, k_{1}\right)+(n-2) F_{\mathrm{Rie}}\left(r, k_{2}\right)\right) \frac{\partial}{\partial r}+\frac{\partial^{2}}{\partial r^{2}}
$$


and $F_{\text {Sas }}, F_{\text {Rie }}$ are the explicit functions defined by

$$
F_{\mathrm{Rie}}(r, k)= \begin{cases}\sqrt{k} \cot \sqrt{k} r & \text { if } k>0 \\ 1 / r & \text { if } k=0 \\ \sqrt{|k|} \operatorname{coth} \sqrt{|k|} r & \text { if } k<0\end{cases}
$$

and

$$
F_{\text {Sas }}(r, k)= \begin{cases}\frac{\sqrt{k}(\sin \sqrt{k} r-\sqrt{k} r \cos \sqrt{k} r)}{2-2 \cos \sqrt{k} r-\sqrt{k} r \sin \sqrt{k} r} & \text { if } k>0, \\ 4 / r & \text { if } k=0, \\ \frac{\sqrt{|k|}(\sqrt{|k|} r \cosh \sqrt{|k|} r-\sinh \sqrt{|k|} r)}{2-2 \cosh \sqrt{|k|} r+\sqrt{|k|} r \sinh \sqrt{|k|} r} & \text { if } k<0 .\end{cases}
$$

For instance, in the case of the 3-dimensional Heisenberg group, which is a Sasakian manifold for which $n=2$ and $k_{1}=0, k_{2}=0$ one can see that

$$
L_{0,0}=\frac{4}{r} \frac{\partial}{\partial r}+\frac{\partial^{2}}{\partial r^{2}} \text {. }
$$

As a consequence, the sub-Riemannian radial part of the sub-Riemannian Brownian motion in the Heisenberg group can be controlled by a 5-dimensional Bessel processes. Note that the dimension 5 here is not too surprising since 5 is the MCP dimension of the Heisenberg group (see [9, Section 3.6] for further comments about the MCP dimension in that context). As a corollary of our comparison theorem, we obtain a Cheng's type estimate for the Dirichlet eigenvalues of sub-Riemannian metric balls, see Section 5.2. In the case $k_{1}=k_{2}=0$, which thus includes the Heisenberg group, our result becomes the following.

Theorem 1.1. Let $\mathbb{M}$ be a sub-Riemannian manifold associated to a Sasakian foliation, with horizontal distribution of dimension $n$, and which satisfies the above comparison result with $k_{1}=k_{2}=0$. For $x_{0} \in \mathbb{M}$ and $R>0$, let $\lambda_{1}\left(B_{0}\left(x_{0}, R\right)\right)$ denote the first Dirichlet eigenvalue of the sub-Riemannian ball $B_{0}\left(x_{0}, R\right)$ and let $\tilde{\lambda}_{1}(d, R)$ denote the first Dirichlet eigenvalue of the Euclidean ball with radius $R$ in $\mathbb{R}^{d}$.

Then, for every $x_{0} \in \mathbb{M}$ and $R>0$

$$
0<\lambda_{1}\left(B_{0}\left(x_{0}, R\right)\right) \leq \tilde{\lambda}_{1}(n+3, R)
$$

The structure of the paper is as follows. In Sections 2, 3 and 4 we consider the general case of totally geodesic Riemannian foliations. After introducing the necessary background on such manifolds in Section 2, we describe the diffusion and drift part of the sub-Riemannian radial process in Section 3. Such a representation allows us to give a criterion for non-explosion of the sub-Riemannian Brownian motion in Section 4, which is more general than previous criteria for stochastic completeness found in [8] and [18]. Finally, we use the sharp comparison theorem available to us for the case of Sasakian manifolds and H-type groups to prove results on the first Dirichlet eigenvalues and on exit times of sub-Riemannian balls in Section 5 .

\section{Preliminaries and assumptions}

In this preliminary section we introduce the geometric framework and recall some of the general sub-Laplacian comparison theorems obtained in [9].

\subsection{Totally geodesic Riemannian foliations and canonical variation}

Let $(\mathrm{M}, g)$ be a complete Riemannian manifold of dimension $n+m$ equipped with a foliation $\mathcal{F}$ with $m$-dimensional leaves. We only consider such manifolds with $n \geq 2$ 
and $m \geq 1$. Let $\mathcal{V}$ be the integrable subbundle tangent to the leaves of $\mathcal{F}$ and write its orthogonal complement with respect to $g$ as $\mathcal{H}$. We will assume that the foliation is Riemannian and with totally geodesic leaves, which is equivalent to the assumptions that

$$
\left(\mathcal{L}_{X} g\right)(Z, Z)=0, \quad\left(\mathcal{L}_{Z} g\right)(X, X)=0, \quad X \in \Gamma^{\infty}(\mathcal{H}), Z \in \Gamma^{\infty}(\mathcal{V})
$$

where $\mathcal{L}$ stands for the Lie derivative. For further details about totally geodesic foliations we refer to [5]. We will also consider the canonical variation $g_{\varepsilon}$ of the Riemannian metric $g$ defined by

$$
g_{\varepsilon}=g_{\mathcal{H}} \oplus \frac{1}{\varepsilon} g_{\mathcal{V}}, \quad g_{\mathcal{H}}=g\left|\mathcal{H}, g_{\mathcal{V}}=g\right| \mathcal{V}, \quad \varepsilon>0
$$

We let $d_{\varepsilon}$ be the Riemannian distance associated to $g_{\varepsilon}$. The limit $\varepsilon \rightarrow 0$ is called the subRiemannian limit. Throughout this paper, we will assume that $\mathcal{H}$ is bracket-generating, i.e. we assume that elements in $\Gamma^{\infty}(\mathcal{H})$ together with all possible iterated brackets of such vector fields span the entire tangent bundle $T \mathrm{IM}$. If this is the case, the limiting distance $d_{0}(x, y)=\lim _{\varepsilon \downarrow 0} d_{\varepsilon}(x, y)$ will always be finite, is called the sub-Riemannian distance and has the following alternative realization. An absolutely continuous curve $\gamma:\left[0, t_{1}\right] \rightarrow \mathbb{M}$ is called horizontal if $\dot{\gamma}(t) \in \mathcal{H}_{\gamma(t)}$ for almost every $t \in\left[0, t_{1}\right]$. It is clear that the length on horizontal curves only depends on $g_{\mathcal{H}}$. The bracket-generating condition ensures that any pair of points can be connected by a horizontal curve and the distance $d_{0}(x, y)$ can be realized as the infimum of the lengths of all horizontal curves connecting the pair of points.

For any fixed $x \in \mathbb{M}$, define $r_{\varepsilon}(y)=d_{\varepsilon}(x, y)$ for any $\varepsilon \geq 0$. We further assume that there are no non-trivial abnormal minimizers for the sub-Riemannian limit; note that this is known to always hold in the Sasakian case [2, Chapt. 8]. The cut locus $\operatorname{Cut}_{\varepsilon}(x)$ is defined such that $y \in \mathbb{M} \backslash \operatorname{Cut}_{\varepsilon}(x)$ if there exists a unique, non-conjugate, lengthminimizing geodesic from $x$ to $y$ relative to $g_{\varepsilon}$. The global cut locus of IM is defined by

$$
\mathrm{Cut}_{\varepsilon}(\mathrm{M})=\left\{(x, y) \in \mathbb{M} \times \mathbb{M}, y \in \mathrm{Cut}_{\varepsilon}(x)\right\} .
$$

So far, the geometry and topology of $\mathrm{Cut}_{0}(\mathrm{M})$ is only poorly understood. However, the following is known:

Lemma 2.1 ([1], [24]). Let $\varepsilon \geq 0$. The following statements hold:

(a) The distance function $x \rightarrow d_{\varepsilon}\left(x_{0}, x\right)$ is locally semi-concave in $\mathbb{M} \backslash\left\{x_{0}\right\}$. In particular, it is twice differentiable almost everywhere.

(b) The set $\mathrm{M} \backslash \mathrm{Cut}_{\varepsilon}\left(x_{0}\right)$ is open and dense in $\mathrm{M}$.

(c) The function $(x, y) \rightarrow d_{\varepsilon}(x, y)^{2}$ is smooth on $\mathrm{M} \times \mathbb{M} \backslash \mathrm{Cut}_{\varepsilon}(\mathrm{M})$.

The following theorem can be found in [10].

Theorem 2.2 ([10]). Let $x, y \in \mathbb{M}$ with $y \notin \mathrm{Cut}_{0}(x)$. Then there exists an open neighbourhood $V$ of $y$ and $\varepsilon^{\prime}>0$ such that $V \cap \mathrm{Cut}_{\varepsilon}(x)=\emptyset$ for all $0 \leq \varepsilon<\varepsilon^{\prime}$. Furthermore, the map

$$
(\varepsilon, z) \mapsto r_{\varepsilon}(z)=d_{\varepsilon}(x, z)
$$

is smooth for $(\varepsilon, z) \in\left[0, \varepsilon^{\prime}\right) \times V$. In particular, we have uniform convergence $r_{\varepsilon} \rightarrow r_{0}$ together with their derivatives of arbitrary order on compact subsets of $\mathrm{M} \backslash \mathrm{Cut}_{0}(x)$. 


\subsection{Sub-Laplacian comparison theorems for the Riemannian approximations}

The Riemannian gradient will be denoted by $\nabla$ and the Riemannian volume by $\mu$ (that is, for $\varepsilon=1$ ) and we write the horizontal gradient as $\nabla_{\mathcal{H}}$, which is the projection of $\nabla$ onto $\mathcal{H}$. The horizontal (or sometimes called sub-Riemannian) Laplacian $\Delta_{\mathcal{H}}$ is the generator of the symmetric closable bilinear form:

$$
\mathcal{E}_{\mathcal{H}}(f, g)=-\int_{\mathbb{M}}\left\langle\nabla_{\mathcal{H}} f, \nabla_{\mathcal{H}} g\right\rangle_{\mathcal{H}} d \mu, \quad f, g \in C_{0}^{\infty}(\mathrm{M}) .
$$

Note that using the volume of any of the Riemannian structures for $\varepsilon>0$ would give the same form and thus the same generator. The hypothesis that $\mathcal{H}$ is bracket generating implies that the horizontal Laplacian $\Delta_{\mathcal{H}}$ is locally subelliptic and the completeness assumption on $g$ implies that $\Delta_{\mathcal{H}}$ is essentially self-adjoint on the space of smooth and compactly supported functions (see for instance [5]). Estimates on $\Delta_{\mathcal{H}} r_{\varepsilon}$ outside of the cut-locus have been obtained in [9] and rely on the control of some tensors associated to a canonical connection (the Bott connection). The exact definition of those tensors is not relevant in the present paper, so for conciseness we omit the details, but refer to [9]. Such tensors were denoted $\mathbf{R i c}_{\mathcal{H}}, \mathbf{J}^{2}$, and $\operatorname{Tr}\left(J_{Z}^{2}\right)$. Throughout the Sections 2,3 and 4 we assume that globally on $\mathbb{M}$, for every $X \in \Gamma^{\infty}(\mathcal{H})$ and $Z \in \Gamma^{\infty}(\mathcal{V})$,

$$
\begin{gathered}
\operatorname{Ric}_{\mathcal{H}}(X, X) \geq \rho_{1}\left(r_{\varepsilon}\right)\|X\|_{\mathcal{H}}^{2}, \\
-\left\langle\mathbf{J}^{2} X, X\right\rangle_{\mathcal{H}} \leq \kappa\left(r_{\varepsilon}\right)\|X\|_{\mathcal{H}}^{2}, \quad-\frac{1}{4} \operatorname{Tr}\left(J_{Z}^{2}\right) \geq \rho_{2}\left(r_{\varepsilon}\right)\|Z\|_{\mathcal{V}}^{2},
\end{gathered}
$$

for some continuous functions $\rho_{1}, \rho_{2}, \kappa$ with $\kappa>0$ and $\rho_{2} \geq 0$. We moreover always assume that the foliation is of Yang-Mills type (see [9]). The main results obtained in [9] under those assumptions are the following:

Theorem 2.3 ([9]). Let $x_{0} \in \mathrm{M}$ be fixed and for $\varepsilon>0$ let $r_{\varepsilon}(x)=d_{\varepsilon}\left(x_{0}, x\right)$. Let $x \in \mathbb{M}$, $x \neq x_{0}$ and $x$ not in the $d_{\varepsilon}$ cut-locus of $x_{0}$. Let $G:\left[0, r_{\varepsilon}(x)\right] \rightarrow \mathbb{R}_{\geq 0}$ be a differentiable function which is positive on $\left(0, r_{\varepsilon}(x)\right]$ and such that $G(0)=0$. We have

$$
\begin{aligned}
\Delta_{\mathcal{H}} r_{\varepsilon}(x) \leq \frac{1}{G\left(r_{\varepsilon}(x)\right)^{2}} \int_{0}^{r_{\varepsilon}(x)}\left(n G^{\prime}(s)^{2}-\right. & {\left[\left(\rho_{1}(s)-\frac{1}{\varepsilon} \kappa(s)\right) \Gamma\left(r_{\varepsilon}\right)(x)\right.} \\
& \left.\left.+\rho_{2}(s) \Gamma^{\mathcal{V}}\left(r_{\varepsilon}\right)(x)\right] G(s)^{2}\right) d s .
\end{aligned}
$$

Corollary 2.4 ([9]). Assume that the functions $\rho_{1}, \kappa, \rho_{2}$ are constant. Denote

$$
\kappa_{\varepsilon}=\min \left(\rho_{1}-\frac{\kappa}{\varepsilon}, \frac{\rho_{2}}{\varepsilon}\right) .
$$

For $x \neq x_{0} \in \mathbb{M}$, not in the $d_{\varepsilon}$ cut-locus of $x_{0}$,

$$
\Delta_{\mathcal{H}} r_{\varepsilon}(x) \leq \begin{cases}\sqrt{n \kappa_{\varepsilon}} \cot \left(\sqrt{\frac{\kappa_{\varepsilon}}{n}} r_{\varepsilon}(x)\right), & \text { if } \kappa_{\varepsilon}>0, \\ \frac{n}{r_{\varepsilon}(x)}, & \text { if } \kappa_{\varepsilon}=0 \\ \sqrt{n\left|\kappa_{\varepsilon}\right|} \operatorname{coth}\left(\sqrt{\frac{\left|\kappa_{\varepsilon}\right|}{n}} r_{\varepsilon}(x)\right), & \text { if } \kappa_{\varepsilon}<0 .\end{cases}
$$

\section{Itô's formula for radial processes}

Let $\left(\left(\xi_{t}\right)_{t \geq 0},\left(\mathbb{P}_{x}\right)_{x \in \mathbb{M}}\right)$ be the subelliptic diffusion process generated by $\Delta_{\mathcal{H}}$ and let $\zeta$ denote its lifetime. We will refer to $\xi$ as the horizontal Brownian motion of the foliation or as the sub-Riemannian Brownian motion (in particular, here our Brownian 
motion is normalized to have $\Delta_{\mathcal{H}}$ as its generator, rather than $\left.\frac{1}{2} \Delta_{\mathcal{H}}\right)$. Note that $\xi$ admits a smooth heat kernel $p_{t}(x, y)$ by the hypoellipticity of $\Delta_{\mathcal{H}}$. Take $x_{0} \in \mathbb{M}$ and set $r_{\varepsilon}(x):=d_{\varepsilon}\left(x_{0}, x\right)$. We denote the open $g_{\varepsilon}$-metric ball of radius $r$ centered at $x$ by $B_{r}^{\varepsilon}(x)$, where $\varepsilon \in[0, \infty)$. The goal of this subsection is to show the following Itô formula for the radial processes $r_{\varepsilon}\left(\xi_{t}\right)$ :

Theorem 3.1. Let $\varepsilon>0$ be fixed. For each $x_{1} \in \mathbb{M}$, if $\xi_{0}=x_{1}$, then there exists a non-decreasing continuous process $l_{t}^{\varepsilon}$ which increases only when $\xi_{t} \in \operatorname{Cut}_{\varepsilon}\left(x_{0}\right)$ and a martingale $\beta_{t}^{\varepsilon}$ on $\mathbb{R}$ with quadratic variation satisfying $d\left\langle\beta^{\varepsilon}\right\rangle \leq 2 d t$ (so in particular $\left.\left\langle\beta^{\varepsilon}\right\rangle_{t} \leq 2 t\right)$ such that

$$
r_{\varepsilon}\left(\xi_{t \wedge \zeta}\right)=r_{\varepsilon}\left(x_{1}\right)+\beta_{t \wedge \zeta}^{\varepsilon}+\int_{0}^{t \wedge \zeta} \Delta_{\mathcal{H}} r_{\varepsilon}\left(\xi_{s}\right) d s-l_{t \wedge \zeta}^{\varepsilon}
$$

holds $\mathbb{P}_{x_{1}}$-almost surely.

We begin the proof with some preparatory lemmas. For the remainder of this section $\varepsilon>0$ will be fixed. The following is the usual Itô formula for a smooth function in a local chart. Let $U$ be an open local chart of $\mathrm{IM}$ in which we have $\Delta_{\mathcal{H}}=\sum_{i=1}^{n} X_{i}^{2}+X_{0}$ with a family of vector fields $X_{0}, X_{1}, \ldots, X_{n}$ on $U$, and let $\xi_{t}$ satisfy the stochastic differential equation

$$
d \xi_{t}=\sum_{i=1}^{n} \sqrt{2} X_{i}\left(\xi_{t}\right) \circ d W_{t}^{i}+X_{0}\left(\xi_{t}\right) d t
$$

where $\left(W_{t}^{1}, \ldots, W_{t}^{n}\right)$ is a Brownian motion on $\mathbb{R}^{n}$. Here $\left(W_{t}^{1}, \ldots, W_{t}^{n}\right)$ is a standard Euclidean Brownian motion, generated by $\frac{1}{2}$ the Laplacian, which explains the factors of $\sqrt{2}$ in the SDE. Let $\tau$ be the first exit time from $U$ of $\xi_{t}$, i.e. $\tau:=\inf \left\{t \geq 0 \mid \xi_{t} \notin U\right\}$.

Lemma 3.2. For any $U$-valued random variable $S$ independent of $W$ and smooth function $f: U \rightarrow \mathbb{R}$, we have

$$
f\left(\xi_{t \wedge \tau}\right)=f(S)+\sum_{i=1}^{n} \sqrt{2} \int_{0}^{t \wedge \tau} X_{i} f\left(\xi_{s}\right) d W_{s}^{i}+\int_{0}^{t \wedge \tau} \Delta_{\mathcal{H}} f\left(\xi_{s}\right) d s
$$

$\mathrm{P}_{S}$-almost surely.

Next we show the following two auxiliary lemmas which concern the occupation time of $\xi_{t}$ at singular points of $r_{\varepsilon}$. The proof is almost the same as the one for Riemannian manifolds, but we give it for completeness.

Lemma 3.3. For $\varepsilon>0$, the set $\left\{t \in[0, \infty) \mid \xi_{t} \in \operatorname{Cut}_{\varepsilon}\left(x_{0}\right)\right\}$ has Lebesgue measure zero $\mathbb{P}_{x_{1}}$-almost surely.

Proof. Since there is a heat kernel $p_{t}$, the law of $\xi_{t}$ under $\mathbb{P}_{x_{1}}$ is absolutely continuous with respect to $\mu$. In addition, we have $\mu\left(\mathrm{Cut}_{\varepsilon}\left(x_{0}\right)\right)=0$ (see [11], for instance). By combining these facts with Fubini's theorem, we obtain

$$
\mathbb{E}_{x_{1}}\left[\int_{0}^{\infty} \mathbb{1}_{\left\{\xi_{s} \in \operatorname{Cut}_{\varepsilon}\left(x_{0}\right)\right\}} d s\right]=\int_{0}^{\infty} \mathbb{P}_{x_{1}}\left\{\xi_{s} \in \operatorname{Cut}_{\varepsilon}\left(x_{0}\right)\right\} d s=0 .
$$

Hence $\int_{0}^{\infty} \mathbb{1}_{\left\{\xi_{s} \in \operatorname{Cut}_{\varepsilon}\left(x_{0}\right)\right\}} d s=0 \mathbb{P}_{x_{1}}$-almost surely, and this is nothing but the conclusion.

Lemma 3.4. $\mathbb{P}_{x_{0}}\left\{\xi_{t}=x_{0}\right.$ at some $\left.t \in(0, \infty)\right\}=0$. 
Proof. We begin with noting that by definition the horizontal Laplacian $\Delta_{\mathcal{H}}$ is the generator of the Dirichlet form:

$$
\mathcal{E}_{\mathcal{H}}(f, g)=-\int_{\mathbb{I}}\left\langle\nabla_{\mathcal{H}} f, \nabla_{\mathcal{H}} g\right\rangle_{\mathcal{H}} d \mu, \quad f, g \in \mathcal{D}\left(\mathcal{E}_{\mathcal{H}}\right) .
$$

Observe also that $\mu\left(B_{r}^{0}\left(x_{0}\right)\right) \leq \mu\left(B_{r}^{\varepsilon}\left(x_{0}\right)\right) \leq C_{\varepsilon} r^{n}$ for small $r>0$. This yields that $\left\{x_{0}\right\}$ is exceptional by applying [25, Theorem 3]. The assumption of the theorem in [25] is satisfied since the Brownian motion $\xi$ is associated with the Dirichlet form $\left(\mathcal{E}_{\mathcal{H}}, \mathcal{D}\left(\mathcal{E}_{\mathcal{H}}\right)\right)$ and the distance $d_{0}$ coincides with the intrinsic distance associated with $\mathcal{E}_{\mathcal{H}}$. Then, by [13, Theorem 4.1.2] and [13, Lemma 4.2.4], $\left\{x_{0}\right\}$ is polar. Thus the claim holds. by

For $\mathbf{R}=\left(R_{1}, R_{2}\right)$ with $R_{1}>R_{2}^{-1}>0$, we define stopping times $T_{R_{i}}^{(i)}(i=1,2)$ and $T_{\mathbf{R}}$

$$
\begin{aligned}
T_{R_{1}}^{(1)} & :=\inf \left\{t \geq 0 \mid r_{\varepsilon}\left(\xi_{t}\right) \geq R_{1}\right\}, \\
T_{R_{2}}^{(2)} & :=\inf \left\{t \geq 0 \mid r_{\varepsilon}\left(\xi_{t}\right) \leq 1 / R_{2}\right\}, \\
T_{\mathbf{R}} & :=T_{R_{1}}^{(1)} \wedge T_{R_{2}}^{(2)},
\end{aligned}
$$

where $a \wedge b=\min \{a, b\}$ for $a, b \in \mathbb{R} \cup\{ \pm \infty\}$. We take $R_{2}$ sufficiently large so that $d_{\varepsilon}\left(x_{0}, \operatorname{Cut}_{\varepsilon}\left(x_{0}\right)\right)>R_{2}^{-1}$ holds, and from now on, we fix $R_{1}$ and $R_{2}$ until the final part of the proof of Theorem 3.1. Let us define a set $A$ by

$$
A:=\left\{\begin{array}{l|l}
(x, y) \in \mathbb{M} \times \mathbb{M} \mid \begin{array}{c}
d_{\varepsilon}\left(x_{0}, x\right) \in\left[R_{2}^{-1}, R_{1}\right], d_{\varepsilon}\left(x_{0}, y\right)=\left(3 R_{2}\right)^{-1} \\
\text { and } d_{\varepsilon}(x, y)=d_{\varepsilon}\left(x_{0}, x\right)-d_{\varepsilon}\left(x_{0}, y\right)
\end{array}
\end{array}\right\} .
$$

Note that $A$ is compact since $d_{\varepsilon}(x, y)$ is continuous as a function of $x$ and $y$. For $(x, y) \in A$, $y$ is on a minimal geodesic joining $x_{0}$ and $x$. In addition, $A \cap \mathrm{Cut}_{\varepsilon}(\mathrm{M})=\emptyset$ holds since we can extend the minimal geodesic from $x$ to $y$ with keeping its minimality. By combining these facts, we conclude

$$
\delta_{1}:=\inf \left\{d_{\varepsilon}\left(x, x^{\prime}\right)+d_{\varepsilon}\left(y, y^{\prime}\right) \mid(x, y) \in A,\left(x^{\prime}, y^{\prime}\right) \in \operatorname{Cut}_{\varepsilon}(\mathrm{M})\right\} \wedge \frac{1}{3 R_{2}}>0
$$

Since we can take $\rho_{1}, \rho_{2}, \kappa$ to be constants on $B_{R_{1}+\left(3 R_{2}\right)^{-1}}^{\varepsilon}\left(x_{0}\right)$, Corollary 2.4 yields that there is a continuous function $V:\left(0, R_{1}+\left(3 R_{2}\right)^{-1}\right) \rightarrow[0, \infty)$ such that

$$
\Delta_{\mathcal{H}} d_{\varepsilon}(x, \cdot)(y) \leq V\left(d_{\varepsilon}(x, y)\right)
$$

holds for $x \in B_{\left(3 R_{2}\right)^{-1}}^{\varepsilon}\left(x_{0}\right)$ and $y \in B_{R_{1}}^{\varepsilon}\left(x_{0}\right) \backslash \operatorname{Cut}_{\varepsilon}(x)$. Set $\bar{V}:=\sup _{\left(3 R_{2}\right)^{-1} \leq r \leq R_{1}} V(r)$.

Lemma 3.5. Let $x \in \operatorname{Cut}_{\varepsilon}\left(x_{0}\right) \cap B_{R_{1}}^{\varepsilon}\left(x_{0}\right)$ and $\delta \in\left(0, \delta_{1}\right)$. Set

$$
\tilde{T}:=\inf \left\{t \geq 0 \mid d_{\varepsilon}\left(x, \xi_{t}\right) \geq \delta\right\} .
$$

Then

$$
\mathbb{E}_{x}\left[d_{\varepsilon}\left(x_{0}, \xi_{t \wedge \tilde{T} \wedge T_{\mathbf{R}}}\right)-d_{\varepsilon}\left(x_{0}, x\right)-\left(t \wedge \tilde{T} \wedge T_{\mathbf{R}}\right) \bar{V}\right] \leq 0 .
$$

Proof. We choose a point $\tilde{x}_{0} \in \mathbb{M}$ as follows: Take a minimal geodesic $\gamma:\left[0, r_{\varepsilon}(x)\right] \rightarrow \mathbb{M}$ from $x_{0}$ to $x$ and define $\tilde{x}_{0}:=\gamma\left(\left(3 R_{2}\right)^{-1}\right)$. Then $\left(x_{0}, \tilde{x}_{0}\right) \in A$ holds by construction. Moreover, by the choice of $\delta>0, \xi_{t} \notin \operatorname{Cut}_{\varepsilon}\left(\tilde{x}_{0}\right)$ for all $t \in\left[0, \tilde{T} \wedge T_{\mathbf{R}}\right)$ under $\mathbb{P}_{x}$. For $y \in \mathbb{M}$, let

$$
\tilde{r}^{+}(y):=d_{\varepsilon}\left(x_{0}, \tilde{x}_{0}\right)+d_{\varepsilon}\left(\tilde{x}_{0}, y\right) .
$$

By the choice of $\tilde{x}_{0}$, we have $\tilde{r}^{+}(x)=d_{\varepsilon}\left(x_{0}, x\right)$. Moreover, by the triangle inequality, $\tilde{r}^{+}(y) \geq d_{\varepsilon}\left(x_{0}, y\right)$ for all $y \in \mathbb{M}$. By the definition of $V$ we have

$$
\Delta_{\mathcal{H}} \tilde{r}^{+}(y) \leq V\left(d_{\varepsilon}\left(\tilde{x}_{0}, y\right)\right)
$$


holds for $y \in B_{R_{1}}\left(x_{0}\right) \backslash \operatorname{Cut}_{\varepsilon}\left(\tilde{x}_{0}\right)$. Note that $V\left(d_{\varepsilon}\left(\tilde{x}_{0}, \xi_{t}\right)\right) \leq \bar{V}$ holds for all $t \in\left[0, \tilde{T} \wedge T_{\mathbf{R}}\right)$ since we have

$$
\frac{1}{3 R_{2}} \leq d_{\varepsilon}\left(x_{0}, \xi_{t}\right)-d_{\varepsilon}\left(x_{0}, \tilde{x}_{0}\right) \leq d_{\varepsilon}\left(\tilde{x}_{0}, \xi_{t}\right) \leq d_{\varepsilon}\left(\tilde{x}_{0}, x\right)+d_{\varepsilon}\left(x, \xi_{t}\right) \leq R_{1}
$$

by the choice of $R_{2}$ and $\delta_{1}$. Therefore

$$
\begin{aligned}
d_{\varepsilon}\left(x_{0}, \xi_{t \wedge \tilde{T} \wedge T_{\mathbf{R}}}\right) & -d_{\varepsilon}\left(x_{0}, x\right)-\left(t \wedge \tilde{T} \wedge T_{\mathbf{R}}\right) \bar{V} \\
& \leq d_{\varepsilon}\left(x_{0}, \xi_{t \wedge \tilde{T} \wedge T_{\mathbf{R}}}\right)-\tilde{r}^{+}\left(\xi_{0}\right)-\int_{0}^{t \wedge \tilde{T} \wedge T_{\mathbf{R}}} V\left(d_{\varepsilon}\left(\tilde{x}_{0}, \xi_{s}\right)\right) d s \\
& \leq \tilde{r}^{+}\left(\xi_{t \wedge \tilde{T} \wedge T_{\mathbf{R}}}\right)-\tilde{r}^{+}\left(\xi_{0}\right)-\int_{0}^{t \wedge \tilde{T} \wedge T_{\mathbf{R}}} \Delta_{\mathcal{H}} \tilde{r}^{+}\left(\xi_{s}\right) d s .
\end{aligned}
$$

Since $\tilde{r}^{+}$is smooth on $B_{\delta}^{\varepsilon}(x)$, the last term is a martingale and thus its expectation is zero. Hence the claim follows.

For $\delta \in\left(0, \delta_{1}\right)$, we define a sequence of stopping times $\left(S_{k}^{\delta}\right)_{m \in \mathbb{N}}$ and $\left(T_{k}^{\delta}\right)_{m \in \mathbb{N}_{0}}$ by

$$
\begin{aligned}
T_{0}^{\delta} & :=0, \\
S_{k}^{\delta} & :=T_{\mathbf{R}} \wedge \inf \left\{t \geq T_{k-1}^{\delta} \mid \xi_{t} \in \operatorname{Cut}_{\varepsilon}\left(x_{0}\right)\right\}, \\
T_{k}^{\delta} & :=T_{\mathbf{R}} \wedge \inf \left\{t \geq S_{k}^{\delta} \mid d_{\varepsilon}\left(\xi_{S_{k}^{\delta}}, \xi_{t}\right) \geq \delta\right\} \wedge\left(S_{k}^{\delta}+\delta\right) .
\end{aligned}
$$

Proposition 3.6. The process $r_{\varepsilon}\left(\xi_{t \wedge T_{\mathbf{R}}}\right)-r_{\varepsilon}\left(\xi_{0}\right)-\left(t \wedge T_{\mathbf{R}}\right) \bar{V}$ is a supermartingale.

Proof. By virtue of the strong Markov property of $\xi$, it suffices to show

$$
\mathbb{E}_{x}\left[r_{\varepsilon}\left(\xi_{t \wedge T_{\mathbf{R}}}\right)-r_{\varepsilon}\left(\xi_{0}\right)-\left(t \wedge T_{\mathbf{R}}\right) \bar{V}\right] \leq 0
$$

for each $0 \leq s<t$. By Lemma 3.5, for all $n \in \mathbb{N}$

$$
\mathbb{E}_{x}\left[r_{\varepsilon}\left(\xi_{t \wedge T_{k}^{\delta}}\right)-r_{\varepsilon}\left(\xi_{t \wedge S_{k}^{\delta}}\right)-\left(t \wedge T_{k}^{\delta}-t \wedge S_{k}^{\delta}\right) \bar{V} \mid \mathcal{F}_{S_{k}^{\delta}}\right] \leq 0
$$

We apply the Itô formula to $r_{\varepsilon}\left(\xi_{t}\right)$ on $t \in\left[T_{k-1}^{\delta}, S_{k}^{\delta}\right]$. By (3.3), we have

$$
\Delta_{\mathcal{H}} r_{\varepsilon}\left(\xi_{t \wedge S_{k}^{\delta}}\right) \leq \bar{V}
$$

for $t>T_{k-1}^{\delta}$. These observations yield

$$
\mathbb{E}_{x}\left[r_{\varepsilon}\left(\xi_{t \wedge S_{k}^{\delta}}\right)-r_{\varepsilon}\left(\xi_{t \wedge T_{k-1}^{\delta}}\right)-\left(t \wedge S_{k}^{\delta}-t \wedge T_{k-1}^{\delta}\right) \bar{V} \mid \mathcal{F}_{T_{k-1}^{\delta}}\right] \leq 0
$$

It remains to show $T_{k}^{\delta} \rightarrow T_{\mathbf{R}}$ as $k \rightarrow \infty$ in order to conclude the claim by the dominated convergence theorem. If $\lim _{k \rightarrow \infty} T_{k}^{\delta}=: T_{\infty}<T_{\mathbf{R}}$ occurs, then $T_{k}^{\delta}-S_{k}^{\delta}>0$ converges to 0 as $k \rightarrow \infty$. In addition, $d\left(\xi_{S_{k}^{\delta}}, \xi_{T_{k}^{\delta}}\right)=\delta$ must hold for infinitely many $k \in \mathbb{N}$. However, the combination of these contradicts the fact that the sample path $\xi_{t}$ is uniformly continuous on $\left[0, T_{\infty}\right]$. Hence $\lim _{k \rightarrow \infty} T_{k}^{\delta}=T_{\mathbf{R}}$ as $k \rightarrow \infty$.

Corollary 3.7. $r_{\varepsilon}\left(\xi_{t \wedge T_{\mathbf{R}}}\right)$ is a semimartingale.

Remark 3.8. Repeating the last argument in the proof of Proposition 3.6 implies that, for each fixed $t>0, t \wedge T_{k}^{\delta}=t \wedge T_{\mathbf{R}}$ holds for sufficiently large $k$ almost surely.

Lemma 3.9. $\lim _{\delta \rightarrow 0} \sum_{k=1}^{\infty}\left|T_{k}^{\delta}-S_{k}^{\delta}\right|=0$ almost surely. 
Proof. For $\delta>0$, let us define random subsets $H_{\delta}$ and $H$ of $\left[0, T_{\mathbf{R}}\right)$ by

$$
\begin{aligned}
H_{\delta} & :=\left\{t \in\left[0, T_{\mathbf{R}}\right) \mid \text { there exists } t^{\prime} \in\left[0, T_{\mathbf{R}}\right) \text { satisfying }\left|t-t^{\prime}\right| \leq \delta \text { and } \xi_{t^{\prime}} \in \mathrm{Cut}_{\varepsilon}\left(x_{0}\right)\right\}, \\
H & :=\left\{t \in\left[0, T_{\mathbf{R}}\right) \mid \xi_{t} \in \operatorname{Cut}_{\varepsilon}\left(x_{0}\right)\right\} .
\end{aligned}
$$

Since the sample path of $\xi$ is continuous and $\mathrm{Cut}_{\varepsilon}\left(x_{0}\right)$ is closed, $H$ is closed and $H=$ $\cap_{\delta>0} H_{\delta}$ holds. By the definition of $S_{k}^{\delta}$ and $T_{k}^{\delta}$, we have

$$
H \subset \bigcup_{m=1}^{\infty}\left[S_{k}^{\delta}, T_{k}^{\delta}\right] \subset H_{\delta}
$$

Hence the monotone convergence theorem yields that, for any $T>0$,

$$
\limsup _{\delta \rightarrow 0} \sum_{m=1}^{\infty}\left|T_{k}^{\delta} \wedge T-S_{k}^{\delta} \wedge T\right| \leq \lim _{\delta \rightarrow 0} \int_{0}^{T} \mathbb{1}_{H_{\delta}}(t) d t=\int_{0}^{T} \mathbb{1}_{H}(t) d t=0
$$

almost surely, where the last equality follows from Lemma 3.3.

Lemma 3.10. Let $U$ be a local chart of $\mathrm{M}$ on which $\Delta_{\mathcal{H}}=\sum_{i=1}^{n} X_{i}^{2}+X_{0}$ holds with a family of vector fields $\left\{X_{i}\right\}_{i=0}^{n}$ on $U$ and $\xi_{t}$ satisfies the stochastic differential equation (3.2) with a Brownian motion $\left(W_{t}^{1}, \ldots, W_{t}^{n}\right)$. Let $\tau_{1}$ and $\tau_{2}$ be stopping times with $\tau_{1}<\tau_{2}$ so that $\xi_{t}$ is in $U$ whenever $t \in\left[\tau_{1}, \tau_{2}\right]$. Then the martingale part of $r_{\varepsilon}\left(\xi_{t \wedge T_{\mathbf{R}} \wedge \tau_{2}}\right)-r_{\varepsilon}\left(\xi_{T_{\mathbf{R}} \wedge \tau_{1}}\right)$ coincides with

$$
\sqrt{2} \sum_{i=1}^{n} \int_{T_{\mathbf{R}} \wedge \tau_{1}}^{t \wedge T_{\mathbf{R}} \wedge \tau_{2}} X_{i} r_{\varepsilon}\left(\xi_{s}\right) d W_{s}^{i}
$$

Moreover, the quadratic variation of the martingale part of $r_{\varepsilon}\left(\xi_{t \wedge T_{\mathbf{R}}}\right)-r_{\varepsilon}\left(\xi_{0}\right)$ is bounded from above by $2 t$.

Proof. We first remark that the integrand $X_{i} r_{\varepsilon}\left(\xi_{s}\right)$ of the Itô stochastic integral (3.4) is well-defined by virtue of Lemma 3.3. Moreover, $\sum_{i=1}^{n}\left|X_{i} r_{\varepsilon}\left(\xi_{s}\right)\right|^{2} \leq 1$ holds for a.e. $s$ $\mathbb{P}_{x_{1}}$-almost surely. By the martingale representation theorem, there exists an $\mathbb{R}^{n}$-valued adapted process $\eta$ such that the martingale part of $r_{\varepsilon}\left(\xi_{t \wedge T_{\mathbf{R}} \wedge \tau_{2}}\right)-r_{\varepsilon}\left(\xi_{T_{\mathbf{R}} \wedge \tau_{1}}\right)$ equals

$$
\sum_{i=1}^{n} \int_{\tau_{1} \wedge T_{\mathbf{R}}}^{t \wedge T_{\mathbf{R}} \wedge \tau_{2}} \eta_{s}^{i} d W_{s}^{i}
$$

Let us define a (local) martingale $N_{t}$ by

$$
N_{t}:=\sum_{i=1}^{n} \int_{T_{\mathbf{R}} \wedge \tau_{1}}^{t \wedge T_{\mathbf{R}} \wedge \tau_{2}} \eta_{s}^{i} d W_{s}^{i}-\sqrt{2} \sum_{i=1}^{n} \int_{T_{\mathbf{R}} \wedge \tau_{1}}^{t \wedge T_{\mathbf{R}} \wedge \tau_{2}} X_{i} r_{\varepsilon}\left(\xi_{s}\right) d W_{s}^{i}
$$

Using the stopping times $S_{k}^{\delta}$ and $T_{k}^{\delta}$, the quadratic variation $\langle N\rangle_{t}$ of $N$ is expressed as follows:

$$
\begin{aligned}
\langle N\rangle_{\tau_{2}}=\sum_{i=1}^{d} \sum_{k=1}^{\infty}( & \int_{T_{k-1}^{\delta} \wedge T_{\mathbf{R}} \wedge \tau_{1}}^{S_{k}^{\delta} \wedge T_{\mathbf{R}} \wedge \tau_{2}}\left|\eta^{i}(t)-\sqrt{2} X_{i} r_{\varepsilon}\left(\xi_{t}\right)\right|^{2} d t \\
& \left.+\int_{S_{k}^{\delta} \wedge T_{\mathbf{R}} \wedge \tau_{1}}^{T_{k}^{\delta} \wedge T_{\mathbf{R}} \wedge \tau_{2}}\left|\eta_{t}^{i}-\sqrt{2} X_{i} r_{\varepsilon}\left(\xi_{t}\right)\right|^{2} d t\right) .
\end{aligned}
$$

Since $\xi_{t} \notin \operatorname{Cut}_{\varepsilon}\left(x_{0}\right)$ if $t \in\left(T_{k-1}^{\delta}, S_{k}^{\delta}\right)$, Lemma 3.2 yields

$$
\int_{T_{k-1}^{\delta} \wedge T_{\mathbf{R}} \wedge \tau_{1}}^{S_{k}^{\delta} \wedge T_{\mathbf{R}} \wedge \tau_{2}}\left|\eta_{t}^{i}-\sqrt{2} X_{i} r_{\varepsilon}\left(\xi_{t}\right)\right|^{2} d t=0
$$


for $k \in \mathbb{N}$ and $i=1, \ldots, n$. For the second term in the right-hand side of (3.5) we have

$$
\sum_{k=1}^{\infty} \int_{S_{k}^{\delta} \wedge T_{\mathbf{R}} \wedge \tau_{1}}^{T_{k}^{\delta} \wedge T_{\mathbf{R}} \wedge \tau_{2}}\left|\eta_{t}^{i}-\sqrt{2} X_{i} r_{\varepsilon}\left(\xi_{t}\right)\right|^{2} d t \leq 2 \int_{\bigcup_{k=1}^{\infty}\left[S_{k}^{\delta} \wedge T_{\mathbf{R}} \wedge \tau_{1}, T_{k}^{\delta} \wedge T_{\mathbf{R}} \wedge \tau_{2}\right]}\left(\left|\eta_{t}\right|^{2}+2\right) d t .
$$

Since $\eta$ is locally square-integrable on $[0, \infty)$ almost surely, Lemma 3.9 yields that the right hand side of (3.6) tends to 0 as $\delta \downarrow 0$. Hence $\langle N\rangle_{\tau_{2}}=0$ and the first assertion follows. The second assertion can be obtained by decomposing $\xi_{t}$ through a sequence of stopping times into sample paths each of which is contained in a local chart and using the strong Markov property.

We are final ready to prove our Itô formula for the radial process.

Proof of Theorem 3.1. On the basis of Corollary 3.7, we denote the martingale part of $r_{\varepsilon}\left(\xi_{t \wedge T_{\mathbf{R}}}\right)$ by $\beta_{t}^{\varepsilon, \mathbf{R}}$. By virtue of (3.3) and Lemma 3.3, the integral of $s \mapsto \Delta_{\mathcal{H}} r_{\varepsilon}\left(\xi_{s}\right)$ on a subset of $\left[0, t \wedge T_{\mathbf{R}}\right]$ is well-defined. Set $I_{\delta}:=\bigcup_{n=1}^{\infty}\left[S_{k}^{\delta}, T_{k}^{\delta}\right]$ and let us define $l_{t}^{\varepsilon, \delta, \mathbf{R}}$ by

$$
\begin{aligned}
l_{t}^{\varepsilon, \delta, \mathbf{R}}:= & -r_{\varepsilon}\left(\xi_{t \wedge T_{\mathbf{R}}}\right)+r_{\varepsilon}\left(\xi_{0}\right)+\beta_{t \wedge T_{\mathbf{R}}}^{\varepsilon, \mathbf{R}} \\
& +\int_{\left[0, t \wedge T_{\mathbf{R}}\right] \backslash I_{\delta}} \Delta_{\mathcal{H}} r_{\varepsilon}\left(\xi_{s}\right) d s+\left|\left[0, t \wedge T_{\mathbf{R}}\right] \cap I_{\delta}\right| \bar{V},
\end{aligned}
$$

where the modulus indicates the Lebesgue measure of the set. By Lemmas 3.2 and 3.10, $l^{\varepsilon, \delta, \mathbf{R}}$ is constant on $\left[0, T_{\mathbf{R}}\right] \backslash I_{\delta}$. Moreover, Proposition 3.6 yields that $l_{t}^{\varepsilon, \delta, \mathbf{R}}$ is nondecreasing in $t$, and in particular $l_{t}^{\varepsilon, \delta, \mathbf{R}} \geq 0$. By Lemma 3.3, $\Delta_{\mathcal{H}} r_{\varepsilon}\left(\xi_{s}\right) \mathbb{1}_{I_{\delta}^{c}}\left(\xi_{s}\right)$ converges to $\Delta_{\mathcal{H}} r_{\varepsilon}\left(\xi_{s}\right)$ as $\delta \downarrow 0$ for a.e. $s \in\left[0, t \wedge T_{\mathbf{R}}\right] \mathbb{P}_{x_{1}}$-a.s. Thus, Fatou's lemma together with (3.3) and Lemma 3.9 yields

$$
0 \leq \limsup _{\delta \downarrow 0} l_{t}^{\varepsilon, \delta, \mathbf{R}} \leq-r_{\varepsilon}\left(\xi_{t \wedge T_{\mathbf{R}}}\right)+r_{\varepsilon}\left(\xi_{0}\right)+\beta_{t \wedge T_{\mathbf{R}}}^{\varepsilon, \mathbf{R}}+\int_{0}^{t \wedge T_{\mathbf{R}}} \Delta_{\mathcal{H}} r_{\varepsilon}\left(\xi_{s}\right) d s .
$$

This inequality and (3.3) ensure that $s \mapsto \Delta_{\mathcal{H}} r_{\varepsilon}\left(\xi_{s}\right)$ belongs to $L^{1}\left(\left[0, t \wedge T_{\mathbf{R}}\right]\right) \mathbb{P}_{x_{1}}$-a.s. Thus, Lemma 3.9 implies that $l_{t}^{\varepsilon, \mathbf{R}}:=\lim _{\delta \downarrow 0} l_{t}^{\varepsilon, \delta, \mathbf{R}}$ exists and

$$
r_{\varepsilon}\left(\xi_{t \wedge T_{\mathbf{R}}}\right)=r_{\varepsilon}\left(\xi_{0}\right)+\beta_{t \wedge T_{\mathbf{R}}}^{\varepsilon, \mathbf{R}}+\int_{0}^{t \wedge T_{\mathbf{R}}} \Delta_{\mathcal{H}} r_{\varepsilon}\left(\xi_{s}\right) d s-l_{t}^{\varepsilon, \mathbf{R}}
$$

holds for all $t \geq 0 \mathbb{P}_{x_{1}}$-a.s. By Lemma 3.4, we can take the limit $R_{2} \rightarrow \infty$ in the last identity in a compatible way. Then the conclusion follows by taking the limit $R_{1} \rightarrow \infty$. Indeed, it is not difficult to see that $l_{t}^{\varepsilon}$ can increase only when $\xi_{t} \in \operatorname{Cut}_{\varepsilon}\left(x_{0}\right)$ from the corresponding property for $l_{t}^{\varepsilon, \delta, \mathbf{R}}$. Moreover, by Lemma 3.10 and the Markov property, we have that $\left\langle\beta^{\varepsilon}\right\rangle_{t}-\left\langle\beta^{\varepsilon}\right\rangle_{s} \leq 2 t-2 s$ for $s<t$, so that $d\left\langle\beta^{\varepsilon}\right\rangle \leq 2 d t$.

\section{Comparison of radial processes and stochastic completeness on general foliations}

\subsection{Comparison of radial processes}

We first recall the definition of a model Riemannian manifold that was introduced by E. Greene and H. Wu, see [15].

Definition 4.1. Let $h:[0,+\infty) \rightarrow(0,+\infty)$ be a smooth function which is positive on $(0,+\infty)$ and such that $h(0)=0, h^{\prime}(0)=1$. Denote $K(r)=-h^{\prime \prime}(r) / h(r)$. The Riemannian manifold $\mathbb{M}_{K}=[0,+\infty) \times \mathbb{S}^{n}$ with Riemannian metric

$$
g=d r^{2}+h(r)^{2} g_{\mathbb{S}^{n}}
$$

is called the Riemannian model space with radial curvature $K(r)$, where $g_{\mathbb{S}^{n}}$ denotes the standard metric on $\mathbb{S}^{n}$. 
As before, we fix a point $x_{0} \in \mathbb{M}$. For $x_{1} \in \mathbb{M}$, we consider the solution of the stochastic differential equation

$$
\tilde{\xi}_{t}^{\varepsilon}=d_{\varepsilon}\left(x_{0}, x_{1}\right)+n \int_{0}^{t} \frac{h^{\prime}\left(\tilde{\xi}_{s}^{\varepsilon}\right)}{h\left(\tilde{\xi}_{s}^{\varepsilon}\right)} d s+\beta_{t}^{\varepsilon}
$$

where $\beta^{\varepsilon}$ is the martingale defined in Theorem 3.1.

Theorem 4.2. Define $\tilde{\xi}_{t}^{\varepsilon}$ as in (4.1) and write $K(r)=-h^{\prime \prime}(r) / h(r)$. Assume that

$$
\min \left\{\rho_{1}(r)-\frac{\kappa(r)}{\varepsilon}, \frac{\rho_{2}(r)}{\varepsilon}\right\} \geq n K(r) .
$$

Let $\left(\left(\xi_{t}\right)_{t \geq 0},\left(\mathbb{P}_{x}\right)_{x \in \mathbb{M}}\right)$ be the horizontal Brownian motion on $\mathbb{M}$ generated by $\Delta_{\mathcal{H}}$.

Then, for any $R \geq d_{\varepsilon}\left(x_{0}, x_{1}\right)$ and any non decreasing function $\phi$ on $[0, R)$,

$$
\mathbb{E}_{x_{1}}\left[\phi\left(d_{\varepsilon}\left(x_{0}, \xi_{t}^{\varepsilon}\right)\right), t<\tau_{R}^{\varepsilon}\right] \leq \mathbb{E}_{x_{1}}\left[\phi\left(\tilde{\xi}_{t}^{\varepsilon}\right), t<\tau_{R}^{\varepsilon}\right]
$$

where $\tau_{R}^{\varepsilon}$ is the hitting time of $R$ by $\tilde{\xi}^{\varepsilon}$.

Proof. It follows from Theorem 3.1 and the Ikeda-Watanabe comparison theorem [21] that for $t<\tau_{R}^{\varepsilon}$, one has $\mathbb{P}_{x_{1}}$ a.s.

$$
d_{\varepsilon}\left(x_{0}, \xi_{t}\right) \leq \tilde{\xi}_{t}^{\varepsilon}
$$

The result follows then immediately.

\subsection{Stochastic completeness criterion}

In this section we prove a general non-explosion criterion for the horizontal Brownian $\left(\xi_{t}\right)_{t \geq 0}$ as a consequence of the sub-Laplacian comparison Theorem 2.3. Recall that the functions $\rho_{1}, \rho_{2}, \kappa$ are defined through the assumptions (2.2) and in particular that $\rho_{2} \geq 0$.

Theorem 4.3. Suppose that for some $\varepsilon>0$ there exists a non-decreasing function $K:[0,+\infty) \rightarrow(0,+\infty)$ satisfying $\int_{0}^{+\infty} K(s)^{-1 / 2} d s=+\infty$ and such that for every $s \geq 0$

$$
\rho_{1}(s)-\frac{\kappa(s)}{\varepsilon} \geq-K(s) .
$$

Then $\left(\xi_{t}\right)_{t \geq 0}$ does not explode.

Proof. Without loss of generality, we can assume that $\xi_{0}=x_{1}$ for some $x_{1} \neq x_{0}$, so that $r_{\varepsilon}\left(\xi_{0}\right)>0$. Let

$$
f(x)=\int_{0}^{x} K(s)^{-1 / 2} d s,
$$

and note that $f$ is continuous, strictly increasing, and proper. Then $f\left(r_{\varepsilon}\left(\xi_{t}\right)\right)$ is semimartingale on $(0, \infty)$, and non-explosion of $f\left(r_{\varepsilon}\left(\xi_{t}\right)\right)$ implies non-explosion of $\xi_{t}$. Note that $f^{\prime}(x)=1 / \sqrt{K(x)}$, which is bounded from above by a positive constant (namely $1 / \sqrt{K(0)})$, and $f^{\prime \prime}(x)=-\frac{K^{\prime}(x)}{2 K^{3 / 2}(x)}$, where $K^{\prime}$ is understood as a positive measure, so that $f^{\prime \prime} \leq 0$ in the sense of distributions.

From Corollary 2.4, we know that

$$
\Delta_{\mathcal{H}} r_{\varepsilon}(x) \leq \sqrt{n K\left(r_{\varepsilon}(x)\right)} \operatorname{coth}\left(\sqrt{K\left(r_{\varepsilon}(x)\right)} r_{\varepsilon}(x)\right)
$$

in the sense of distributions. Let $F_{t}$ be the semi-martingale on $(0, \infty)$ given by the solution to the SDE

$$
d F_{t}=\frac{1}{\sqrt{K\left(r_{\varepsilon}\left(\xi_{t}\right)\right)}} d \beta_{t}^{\varepsilon}+\sqrt{n} \operatorname{coth}\left(\sqrt{K\left(f^{-1}\left(F_{t}\right)\right)} f^{-1}\left(F_{t}\right)\right) d t ; \quad F_{0}=f\left(r_{\varepsilon}\left(\xi_{0}\right)\right),
$$


where $\beta_{t}^{\varepsilon}$ is the martingale part of $r_{\varepsilon}\left(\xi_{t}\right)$ and $f^{-1}$ is the inverse of $f(x)$. Then since $-f$ is convex, we can apply the (extended) Itô rule, starting with the decomposition of $r_{\varepsilon}\left(\xi_{t}\right)$ from Theorem 3.1, (and standard SDE comparison) to see that $f\left(r_{\varepsilon}\left(\xi_{t}\right)\right)$ is bounded from above by $F_{t}$. Now the diffusivity of the above SDE satisfied by $F_{t}$ is bounded, and the drift is bounded when $F_{t} \geq 1$, so $F_{t}$ cannot explode. It follows that $f\left(r_{\varepsilon}\left(\xi_{t}\right)\right)$ and hence $\xi_{t}$ also cannot explode, completing the proof.

In particular, this implies that a natural type of quadratic curvature decay condition implies stochastic completeness, analogously to the well-known Riemannian situation. More precisely, we have the following.

Corollary 4.4. Suppose that for some $\varepsilon>0$, there exists $c_{1}>0$ such that

$$
\rho_{1}(s)-\frac{\kappa(s)}{\varepsilon} \geq-\left(c_{1}^{2} s^{2}+c_{1}\right)
$$

holds for all $s>0$. Then $\left(\xi_{t}\right)_{t \geq 0}$ does not explode.

\section{Comparison theorems for the radial processes on Sasakian manifolds}

In this section we study in more detail specific foliations on which the theory can be pushed further. The foliations we consider are called Sasakian foliations. Those are well-studied co-dimension one totally geodesic foliations with additional structure described in [9, Section 3] (we also for instance refer to [4] and the references inside for further details).

\subsection{Comparison of radial processes}

In this section, we use the sub-Laplacian comparison theorem on Sasakian manifolds foliations in [9] to get estimates for radial parts of the horizontal Brownian motion. In the Riemannian case the method we use is due to K. Ichihara [20]. In the case where the Ricci curvature is bounded from below by a constant, the method yields the sharp Cheeger-Yau lower bound [12] for the heat kernel.

We first briefly recall the sub-Laplacian comparison theorem proved in [9] to which we refer for further details. Recall the comparison functions $F_{\text {Rie }}$ and $F_{\text {Sas }}$ given as in respectively (1.1) and (1.2).

Theorem 5.1 ([9]). Let $(\mathbb{M}, \mathcal{F}, g)$ be a Sasakian foliation with sub-Riemannian distance $d_{0}$. Let $x_{0} \in \mathbb{M}$ and define $r_{0}(x)=d\left(x_{0}, x\right)$. Assume that for some $k_{1}, k_{2} \in \mathbb{R}$

$$
\mathbf{K}_{\mathcal{H}, J}(v, v) \geq k_{1}, \quad \operatorname{Ric}_{\mathcal{H}, J^{\perp}}(v, v) \geq(n-2) k_{2}, \quad v \in \mathcal{H},\|v\|_{g}=1 .
$$

Then outside of the $d_{0}$ cut-locus of $x_{0}$ and globally on $\mathbb{M}$ in the sense of distributions,

$$
\Delta_{\mathcal{H}} r_{0} \leq F_{\text {Sas }}\left(r, k_{1}\right)+(n-2) F_{\text {Rie }}\left(r, k_{2}\right) .
$$

The tensors $\mathbf{K}_{\mathcal{H}, J}$ and $\mathbf{R i c}_{\mathcal{H}, J^{\perp}}$ are defined in [9]. We omit here their definition for conciseness since they will not be relevant in our analysis except as criteria to get the sub-Laplacian comparison theorem.

As before, we fix a point $x_{0} \in \mathbb{M}$. For $x_{1} \in \mathbb{M}$, we consider the solution of the stochastic differential equation

$$
\tilde{\xi}_{t}=d_{0}\left(x_{0}, x_{1}\right)+\int_{0}^{t}\left(F_{\text {Sas }}\left(\tilde{\xi}_{s}, k_{1}\right)+(n-2) F_{\text {Rie }}\left(\tilde{\xi}_{s}, k_{2}\right)\right) d s+\sqrt{2} \beta_{t}
$$

where $\beta$ is a standard Brownian motion under $\mathbb{P}_{x_{1}}$. 
Theorem 5.2. Let $(\mathrm{M}, \mathcal{F}, g)$ be a Sasakian foliation with sub-Riemannian distance $d_{0}$. Assume that for some $k_{1}, k_{2} \in \mathbb{R}$,

$$
\mathbf{K}_{\mathcal{H}, J}(v, v) \geq k_{1}, \quad \operatorname{Ric}_{\mathcal{H}, J^{\perp}}(v, v) \geq(n-2) k_{2}, \quad v \in \mathcal{H},\|v\|_{g}=1 .
$$

Let $\left(\left(\xi_{t}\right)_{t \geq 0},\left(\mathbb{P}_{x}\right)_{x \in \mathbb{M}}\right)$ be the horizontal Brownian motion on $\mathbb{M}$ generated by $\Delta_{\mathcal{H}}$. Then, for $x_{1} \in \mathrm{M}, R>0$, and $s \leq R$

$$
\mathbb{P}_{x_{1}}\left\{d_{0}\left(x_{0}, \xi_{t}\right)<s, t \leq \tau_{R}\right\} \geq \mathbb{P}_{x_{1}}\left\{\tilde{\xi}_{t}<s, t \leq \tilde{\tau}_{R}\right\}
$$

where $\tau_{R}$ is the hitting time of the $d_{0}$-geodesic ball in $\mathrm{IM}$ with center $x_{0}$ and radius $R$ and $\tilde{\tau}_{R}$ the hitting time of the level $R$ by $\tilde{\xi}$.

Proof. Let $\phi$ be a non-increasing function on $[0, R]$ which is compactly supported on $[0, s]$. We set

$$
u(t, x)=\mathbb{E}_{x}\left[\phi\left(d_{0}\left(x_{0}, \xi_{t}\right)\right), t \leq \tau_{R}\right]
$$

and

We then have

$$
u_{0}(t, r)=\mathbb{E}_{r}\left[\phi\left(\tilde{\xi}_{t}\right), t \leq \tilde{\tau}_{R}\right]
$$

$$
\left\{\begin{array}{l}
u \in C^{\infty}\left((0,+\infty) \times B_{0}\left(x_{0}, R\right)\right) \\
\frac{\partial u}{\partial t}=\Delta_{\mathcal{H}} u \\
u(0, x)=\phi\left(d_{0}\left(x_{0}, x\right)\right), \quad u(t, x)=0 \text { if } x \in \partial B_{0}\left(x_{0}, R\right)
\end{array}\right.
$$

and

$$
\left\{\begin{array}{l}
u_{0} \in C^{\infty}((0,+\infty) \times[0, R)) \\
\frac{\partial u_{0}}{\partial t}=L u_{0} \\
u_{0}(0, r)=\phi(r), \quad u_{0}(t, r)=0 \text { if } r=R
\end{array}\right.
$$

where

$$
L=\left(F_{\text {Sas }}\left(r, k_{1}\right)+(n-2) F_{\text {Rie }}\left(r, k_{2}\right)\right) \frac{\partial}{\partial r}+\frac{\partial^{2}}{\partial r^{2}} .
$$

Similarly to Lemma 2.1 in [20], $u_{0}(t, r)$ is non-increasing in $r$.

For $t \geq 0, x \in B_{0}\left(x_{0}, R\right)$ denote $v(t, x)=u_{0}\left(t, d_{0}\left(x_{0}, x\right)\right)$. For $x \in B_{0}\left(x_{0}, R\right) \backslash \mathrm{Cut}_{x_{0}}(\mathrm{M})$, one has then from Theorem 5.1,

$$
\begin{aligned}
& \Delta_{\mathcal{H}} v(t, x)=\frac{\partial^{2} u_{0}}{\partial r^{2}}\left(t, d_{0}\left(x_{0}, x\right)\right)+\Delta_{\mathcal{H}} r_{0}(x) \frac{\partial u_{0}}{\partial r}\left(t, d_{0}\left(x_{0}, x\right)\right) \\
& \geq \frac{\partial^{2} u_{0}}{\partial r^{2}}\left(t, d_{0}\left(x_{0}, x\right)\right)+\left(F_{\text {Sas }}\left(d_{0}\left(x_{0}, x\right), k_{1}\right)+(n-2) F_{\text {Rie }}\left(d_{0}\left(x_{0}, x\right), k_{2}\right)\right) \frac{\partial u_{0}}{\partial r}\left(t, d_{0}\left(x_{0}, x\right)\right) \\
& \geq L u_{0}\left(t, d_{0}\left(x_{0}, x\right)\right)=\frac{\partial u_{0}}{\partial t}\left(t, d_{0}\left(x_{0}, x\right)\right)=\frac{\partial v}{\partial t}(t, x) .
\end{aligned}
$$

Therefore, by using the semi-concavity of the sub-Riemannian distance and arguing as in the proof of Theorem 10.1 in [3], one deduces that in the sense of distributions one has for $t \geq 0, x \in B_{0}\left(x_{0}, R\right)$,

Since

$$
\frac{\partial v}{\partial t}(t, x) \leq \Delta_{\mathcal{H}} v(t, x)
$$

$$
v(0, x)=\phi\left(d_{0}\left(x_{0}, x\right)\right), \quad v(t, x)=0 \text { if } x \in \partial B_{0}\left(x_{0}, R\right),
$$

a standard parabolic comparison theorem yields

$$
v(t, x) \leq u(t, x)
$$

Taking $\phi=1$ on $[0, s)$ yields the conclusion. 
As a first corollary we deduce exit time estimates:

Corollary 5.3. Under the same assumptions as in Theorem 5.2 one has

$$
\mathbb{E}_{x_{1}}\left[\tau_{R}\right] \geq \mathbb{E}_{x_{1}}\left[\tilde{\tau}_{R}\right]
$$

As a second corollary we deduce estimates for the integrals of the Dirichlet heat kernel on sub-Riemannian balls:

Corollary 5.4. Let $(\mathbb{M}, \mathcal{F}, g)$ be a Sasakian foliation. Assume that for some $k_{1}, k_{2} \in \mathbb{R}$

$$
\mathbf{K}_{\mathcal{H}, J}(v, v) \geq k_{1}, \quad \mathbf{R i c}_{\mathcal{H}, J^{\perp}}(v, v) \geq(n-2) k_{2}, \quad v \in \mathcal{H},\|v\|_{g}=1 .
$$

Fix $R>0$ and let $\left(\left(\xi_{t}^{R}\right)_{t \geq 0},\left(\mathbb{P}_{x}\right)_{x \in B_{0}\left(x_{0}, R\right)}\right)$ be the horizontal Brownian motion on $B_{0}\left(x_{0}, R\right)$ generated by $\Delta_{\mathcal{H}}$ and with Dirichlet boundary condition. Write its heat kernel with respect to the Riemannian volume measure $\mu$ as $p^{R}(t, x, y)$. Let $q^{R}\left(t, r_{1}, r_{2}\right)$ be the heat kernel with respect to the Lebesgue measure on $[0, R]$ of the diffusion with generator

$$
L_{k_{1}, k_{2}}=\left(F_{\text {Sas }}\left(r, k_{1}\right)+(n-2) F_{\text {Rie }}\left(r, k_{2}\right)\right) \frac{\partial}{\partial r}+\frac{\partial^{2}}{\partial r^{2}}
$$

with Dirichlet boundary condition at $R$. Then, for every $s<R, t>0$ and $x_{1} \in B_{0}\left(x_{0}, s\right)$

$$
\int_{B_{0}\left(x_{0}, s\right)} p^{R}\left(t, x_{1}, y\right) d \mu(y) \geq \int_{0}^{s} q^{R}\left(t, d\left(x_{0}, x_{1}\right), r\right) d r .
$$

It is interesting to note that Corollary 5.4 does not yield a lower bound for the heat kernel $p^{R}$ as one could at first expect. Indeed, when $s$ is small the volume of $B_{0}\left(x_{0}, s\right)$ has order $s^{n+2}$, because $n+2$ is the Hausdorff dimension of $\mathbb{M}$ for the metric $d_{0}$. On the other hand, one can directly check that $\int_{0}^{s} q^{R}\left(t, d\left(x_{0}, x_{1}\right), r\right) d r$ has order $s^{n+3}$. This discrepancy is due to the fact that on sub-Riemannian manifolds, the measure contraction dimension is larger than the Hausdorff dimension.

\subsection{Application to Dirichlet eigenvalue estimates}

We now use the comparison theorem of the previous subsection to deduce estimates on the first Dirichlet eigenvalue of the sub-Riemannian balls. This is the sub-Riemannian version of the well-known Cheng's comparison theorem in Riemannian geometry.

For simplicity, we start with the non-negative curvature case $k_{1}=k_{2}=0$.

Let $(\mathbb{M}, \mathcal{F}, g)$ be a Sasakian foliation with sub-Riemannian distance $d_{0}$. Assume that:

$$
\mathbf{K}_{\mathcal{H}, J}(v, v) \geq 0, \quad \mathbf{R i c}_{\mathcal{H}, J^{\perp}}(v, v) \geq 0, \quad v \in \mathcal{H},\|v\|_{g}=1 .
$$

In that case, the one-dimensional diffusion with respect to which we do the comparison is very simple since

$$
L_{0,0}=\frac{n+2}{r} \frac{\partial}{\partial r}+\frac{\partial^{2}}{\partial r^{2}}
$$

which is a Bessel diffusion of dimension $n+3$. We recall that $n$ is the dimension of the horizontal distribution.

Theorem 5.5. Assume $k_{1}=k_{2}=0$. For $x_{0} \in \mathbb{M}$ and $R>0$, let $\lambda_{1}\left(B_{0}\left(x_{0}, R\right)\right)$ denote the first Dirichlet eigenvalue of the sub-Riemannian ball $B_{0}\left(x_{0}, R\right)$ and let $\tilde{\lambda}_{1}(d, R)$ denote the first Dirichlet eigenvalue of Euclidean ball with radius $R$ in $\mathbb{R}^{d}$. Then, for every $x_{0} \in \mathrm{M}$ and $R>0$

$$
\lambda_{1}\left(B_{0}\left(x_{0}, R\right)\right) \leq \tilde{\lambda}_{1}(n+3, R)
$$


Proof. From spectral theory, one has

$$
p^{R}\left(t, x_{1}, y\right)=\sum_{k=1}^{+\infty} e^{-\lambda_{k} t} \phi_{k}\left(x_{1}\right) \phi_{k}(y)
$$

where the $\lambda_{k}$ 's are the Dirichlet eigenvalues of $B_{0}\left(x_{0}, R\right)$ and the $\phi_{k}$ 's the eigenfunctions. One has similarly

$$
q^{R}\left(t, r_{0}, r\right)=r^{n+2} \sum_{k=1}^{+\infty} e^{-\tilde{\lambda}_{k} t} \tilde{\phi}_{k}\left(r_{0}\right) \tilde{\phi}_{k}(r)
$$

Thus, from Corollary 5.4, when $t \rightarrow+\infty$ one must have $\lambda_{1} \leq \tilde{\lambda}_{1}$.

For general $k_{1}, k_{2}$ one can similarly prove the following theorem:

Theorem 5.6. For $x_{0} \in \mathbb{M}$ and $R>0$, let $\lambda_{1}\left(B_{0}\left(x_{0}, R\right)\right)$ denote the first Dirichlet eigenvalue of the sub-Riemannian ball $B_{0}\left(x_{0}, R\right)$ and let $\tilde{\lambda}_{1}\left(n, k_{1}, k_{2}, R\right)$ denote the first Dirichlet eigenvalue of the operator

$$
L_{k_{1}, k_{2}}=\left(F_{\mathrm{Sas}}\left(r, k_{1}\right)+(n-2) F_{\mathrm{Rie}}\left(r, k_{2}\right)\right) \frac{\partial}{\partial r}+\frac{\partial^{2}}{\partial r^{2}}
$$

on the interval $[0, R]$ with Dirichlet boundary condition at $R$. Then, for every $x_{0} \in \mathbb{M}$ and $R>0$

$$
\lambda_{1}\left(B_{0}\left(x_{0}, R\right)\right) \leq \tilde{\lambda}_{1}\left(n, k_{1}, k_{2}, R\right)
$$

\subsection{Large time behavior and law of iterated logarithm for the radial processes}

In this section we study large time behaviour of the radial process in negative curvature.

Proposition 5.7. Let $(\mathrm{M}, \mathcal{F}, g)$ be a Sasakian foliation. Assume that for some $k_{1}, k_{2} \leq 0$

$$
\mathbf{K}_{\mathcal{H}, J}(v, v) \geq k_{1}, \quad \mathbf{R i c}_{\mathcal{H}, J^{\perp}}(v, v) \geq(n-2) k_{2}, \quad v \in \mathcal{H},\|v\|_{g}=1 .
$$

Let $\left(\left(\xi_{t}\right)_{t \geq 0},\left(\mathbb{P}_{x}\right)_{x \in \mathbb{M}}\right)$ be the sub-Riemannian Brownian motion generated by $\Delta_{\mathcal{H}}$. Then for every $x_{0}, x_{1} \in \mathbb{M}$,

$$
\mathbb{P}_{x_{1}}\left(\limsup _{t \rightarrow+\infty} \frac{d_{0}\left(x_{0}, \xi_{t}\right)}{t} \leq(n-2) \sqrt{\left|k_{2}\right|}+\sqrt{\left|k_{1}\right|}\right)=1
$$

Proof. We note that when $k_{1}, k_{2} \leq 0$, the diffusion with generator

$$
L_{k_{1}, k_{2}}=\left(F_{\mathrm{Sas}}\left(r, k_{1}\right)+(n-2) F_{\mathrm{Rie}}\left(r, k_{2}\right)\right) \frac{\partial}{\partial r}+\frac{\partial^{2}}{\partial r^{2}}
$$

is transient and that

$$
\left.\lim _{r \rightarrow+\infty} F_{\text {Sas }}\left(r, k_{1}\right)=\sqrt{\left|k_{1}\right|}, \quad \lim _{r \rightarrow+\infty} F_{\text {Rie }}\left(r, k_{2}\right)\right)=\sqrt{\left|k_{2}\right|} .
$$

The result follows then from similar arguments as in Example 2.1 in [20].

When $k_{1}, k_{2}=0$, the above estimate can be refined and we obtain a law of iterated logarithm.

Proposition 5.8. Let $(\mathbb{M}, \mathcal{F}, g)$ be a Sasakian foliation. Assume that

$$
\mathbf{K}_{\mathcal{H}, J}(v, v) \geq 0, \quad \operatorname{Ric}_{\mathcal{H}, J^{\perp}}(v, v) \geq 0, \quad v \in \mathcal{H},\|v\|_{g}=1 .
$$

Let $\left(\left(\xi_{t}\right)_{t \geq 0},\left(\mathbb{P}_{x}\right)_{x \in \mathbb{M}}\right)$ be the sub-Riemannian Brownian motion generated by $\Delta_{\mathcal{H}}$. Then for every $x_{0}, x_{1} \in \mathbb{M}$,

$$
\mathbb{P}_{x_{1}}\left(\limsup _{t \rightarrow+\infty} \frac{d_{0}\left(x_{0}, \xi_{t}\right)}{\sqrt{2 t \ln \ln t}} \leq 1\right)=1
$$

Proof. The proof is similar to the one of Theorem 3.1 in [20], so we omit the details for conciseness. 


\subsection{Some extensions: H-type groups}

In the recent work [10] sub-Laplacian comparison theorems have been obtained in a more general setting than Sasakian, the setting of H-type sub-Riemannian spaces. In particular, this was proved that if $(\mathrm{M}, \mathcal{F})$ is the totally geodesic foliation on a H-type group, then one has

$$
\Delta_{\mathcal{H}} r_{0} \leq \frac{n+3 m-1}{r_{0}}
$$

classically outside of the cut-locus and globally in the sense of distributions, where as usual $n$ denotes the dimension of the horizontal bundle and $m$ denotes the codimension of this horizontal bundle. In that setting, all the results obtained in this section may be generalized with identical proofs. In particular, one obtains the following Cheng's type theorem for the Dirichlet eigenvalues of sub-Riemannian balls in H-type groups.

Theorem 5.9. Assume that $\mathrm{M}$ is an H-type group. For $x_{0} \in \mathbb{M}$ and $R>0$, let $\lambda_{1}\left(B_{0}\left(x_{0}, R\right)\right)$ denote the first Dirichlet eigenvalue of the sub-Riemannian ball $B_{0}\left(x_{0}, R\right)$ and let $\tilde{\lambda}_{1}(d, R)$ denote the first Dirichlet eigenvalue of Euclidean ball with radius $R$ in $\mathbb{R}^{d}$. Then, for every $x_{0} \in \mathbb{M}$ and $R>0$,

$$
\lambda_{1}\left(B_{0}\left(x_{0}, R\right)\right) \leq \tilde{\lambda}_{1}(n+3 m, R) .
$$

Note that in contrast to the Riemannian case, here the comparison diffusion is not realized as the radial process of a sub-Riemannian manifold (and in particular, it is not the radial process of the Heisenberg group, which fails to be a diffusion), and thus there is no reason to suspect that the bound is sharp.

\section{References}

[1] A. Agrachev, Any sub-Riemannian metric has points of smoothness, Dokl. Akad. Nauk 424 (2009), no. 3, 295-298. MR-2513150

[2] A. Agrachev, D. Barilari, and U. Boscain, A comprehensive introduction to sub-Riemannian geometry, Cambridge Studies in Advanced Mathematics, vol. 181, Cambridge University Press, Cambridge, 2020, From the Hamiltonian viewpoint, With an appendix by Igor Zelenko. MR-3971262

[3] A. Agrachev and P. W. Y. Lee, Bishop and Laplacian comparison theorems on three-dimensional contact sub-Riemannian manifolds with symmetry, J. Geom. Anal. 25 (2015), no. 1, 512-535. MR-3299291

[4] E. Barletta and S. Dragomir, Jacobi fields of the Tanaka-Webster connection on Sasakian manifolds, Kodai Math. J. 29 (2006), no. 3, 406-454. MR-2278775

[5] F. Baudoin, Sub-Laplacians and hypoelliptic operators on totally geodesic Riemannian foliations, Geometry, analysis and dynamics on sub-Riemannian manifolds. Vol. 1, EMS Ser. Lect. Math., Eur. Math. Soc., Zürich, 2016, pp. 259-321. MR-3587668

[6] F. Baudoin, Stochastic analysis on sub-Riemannian manifolds with transverse symmetries, Ann. Probab. 45 (2017), no. 1, 56-81. MR-3601645

[7] F. Baudoin, Q. Feng, and M. Gordina, Integration by parts and quasi-invariance for the horizontal Wiener measure on foliated compact manifolds, J. Funct. Anal. 277 (2019), no. 5, 1362-1422. MR-3969194

[8] F. Baudoin and N. Garofalo, Curvature-dimension inequalities and Ricci lower bounds for sub-Riemannian manifolds with transverse symmetries, J. Eur. Math. Soc. (JEMS) 19 (2017), no. 1, 151-219. MR-3584561

[9] F. Baudoin, E. Grong, K. Kuwada, and A. Thalmaier, Sub-Laplacian comparison theorems on totally geodesic Riemannian foliations, Calc. Var. Partial Differential Equations 58 (2019), no. 4, Paper No. 130, 38. MR-3978951

[10] F. Baudoin, E. Grong, G. Molino, and L. Rizzi, Comparison theorems on H-type sub-Riemannian manifolds, arXiv:1909.03532 (2019). 
Radial processes for sub-Riemannian Brownian motions

[11] I. Chavel, Riemannian geometry-a modern introduction, Cambridge Tracts in Mathematics, vol. 108, Cambridge University Press, Cambridge, 1993. MR-1271141

[12] J. Cheeger and S. T. Yau, A lower bound for the heat kernel, Comm. Pure Appl. Math. 34 (1981), no. 4, 465-480. MR-0615626

[13] M. Fukushima, Y. Oshima, and M. Takeda, Dirichlet forms and symmetric Markov processes, extended ed., De Gruyter Studies in Mathematics, vol. 19, Walter de Gruyter \& Co., Berlin, 2011. MR-2778606

[14] M. Gordina and T. Laetsch, A convergence to Brownian motion on sub-Riemannian manifolds, Trans. Amer. Math. Soc. 369 (2017), no. 9, 6263-6278. MR-3660220

[15] R. E. Greene and H. Wu, Function theory on manifolds which possess a pole, Lecture Notes in Mathematics, vol. 699, Springer, Berlin, 1979. MR-0521983

[16] E. Grong and A. Thalmaier, Curvature-dimension inequalities on sub-Riemannian manifolds obtained from Riemannian foliations: part I, Math. Z. 282 (2016), no. 1-2, 99-130. MR3448376

[17] E. Grong and A. Thalmaier, Curvature-dimension inequalities on sub-Riemannian manifolds obtained from Riemannian foliations: part II, Math. Z. 282 (2016), no. 1-2, 131-164. MR3448377

[18] E. Grong and A. Thalmaier, Stochastic completeness and gradient representations for subRiemannian manifolds, Potential Anal. 51 (2019), no. 2, 219-254. MR-3983506

[19] E. P. Hsu, Stochastic analysis on manifolds, Graduate Studies in Mathematics, vol. 38, American Mathematical Society, Providence, RI, 2002. MR-1882015

[20] K. Ichihara, Comparison theorems for Brownian motions on Riemannian manifolds and their applications, J. Multivariate Anal. 24 (1988), no. 2, 177-188. MR-0926351

[21] N. Ikeda and S. Watanabe, A comparison theorem for solutions of stochastic differential equations and its applications, Osaka Math. J. 14 (1977), no. 3, 619-633. MR-0471082

[22] W. S. Kendall, The radial part of Brownian motion on a manifold: a semimartingale property, Ann. Probab. 15 (1987), no. 4, 1491-1500. MR-0905343

[23] P. W. Y. Lee, Bishop and Laplacian comparison theorems on three-dimensional contact subRiemannian manifolds with symmetry, J. Geom. Anal. 25 (2015), no. 1, 512-535. MR-3299291

[24] L. Rifford and E. Trélat, Morse-Sard type results in sub-Riemannian geometry, Math. Ann. 332 (2005), no. 1, 145-159. MR-2139255

[25] K.-T. Sturm, Sharp estimates for capacities and applications to symmetric diffusions, Probab. Theory Related Fields 103 (1995), no. 1, 73-89. MR-1347171

[26] A. Thalmaier, Geometry of subelliptic diffusions, Geometry, analysis and dynamics on subRiemannian manifolds. Vol. II, EMS Ser. Lect. Math., Eur. Math. Soc., Zürich, 2016, pp. 85169. MR-3642644

Acknowledgments. We thank the anonymous referees for their careful reading and feedback which improved the paper - in particular, for suggesting an improvement to Theorem 4.3. 


\section{Electronic Journal of Probability Electronic Communications in Probability}

\section{Advantages of publishing in EJP-ECP}

- Very high standards

- Free for authors, free for readers

- Quick publication (no backlog)

- Secure publication $\left(\mathrm{LOCKSS}^{1}\right)$

- Easy interface (EJMS²)

\section{Economical model of EJP-ECP}

- Non profit, sponsored by $\mathrm{IMS}^{3}, \mathrm{BS}^{4}$, ProjectEuclid ${ }^{5}$

- Purely electronic

\section{Help keep the journal free and vigorous}

- Donate to the IMS open access fund ${ }^{6}$ (click here to donate!)

- Submit your best articles to EJP-ECP

- Choose EJP-ECP over for-profit journals

\footnotetext{
${ }^{1}$ LOCKSS: Lots of Copies Keep Stuff Safe http://www. lockss.org/

${ }^{2}$ EJMS: Electronic Journal Management System http://www.vtex.lt/en/ejms.html

${ }^{3}$ IMS: Institute of Mathematical Statistics http://www.imstat.org/

${ }^{4}$ BS: Bernoulli Society http://www. bernoulli-society.org/

${ }^{5}$ Project Euclid: https://projecteuclid.org/

${ }^{6}$ IMS Open Access Fund: http://www.imstat.org/publications/open.htm
} 